Open Science at Liberal Arts Colleges

\author{
Kristin A. Lane \\ Bard College \\ Benjamin Le \\ Haverford College \\ Julie A. Woodzicka \\ Washington and Lee University \\ Jerusha B. Detweiler-Bedell \\ Lewis \& Clark College \\ Brian Detweiler-Bedell \\ Lewis \& Clark College
}

Author Note

We thank Dayveliz Hernandez Muztafa for her helpful comments on this manuscript and assistance with its preparation. 


\begin{abstract}
Adopting and sustaining open science practices is accompanied by particular opportunities and challenges for faculty at small liberal arts colleges (SLACs). Their predominantly undergraduate student body, small size, limited resources, substantial teaching responsibilities, and focus on intensive faculty-student interactions make it difficult to normalize open science at SLACs. However, given the unique synergy between teaching and research at SLACs, many of these practices are well-suited for work with undergraduate psychology students. In addition, the opportunities for collaboration afforded by the open science community may be especially attractive for those doing research at SLACs. In this paper, we offer suggestions for how open science can further grow and flourish among faculty who work closely with undergraduates, both in classrooms and in labs. We also discuss how to encourage professional development and transform institutional culture around open science practices. Most importantly, this paper serves as an invitation to SLAC psychology faculty to participate in the open science community.
\end{abstract}




\section{Open Science at Liberal Arts Colleges}

The past decade in psychology has been described as a "crisis" (Fanelli, 2018), "cultural revolution" (Vazire, 2018), and a "renaissance" (Nelson, Simmons, \& Simonsohn, 2018). While these terms differ in their degree of inherent pessimism or optimism, all capture psychological scientists' march toward open science. As faculty at Small Liberal Arts Colleges (SLACS), we suggest that the structure and mission of these unique learning environments result in singular opportunities and challenges for faculty who incorporate open science into their teaching and research.

\section{The Liberal Arts Context}

Exceptional undergraduate education is the primary focus at SLACs, where faculty prioritize students as they engage in their scholarly work and institutional service. The integration of teaching and research characterize the SLAC experience, with meaningful incorporation of students in research central to the institutional mission. Juggling and integrating teaching, research, and service offer great challenges and rewards. Given constraints on time and the aim to integrate research and teaching, faculty must engage undergraduates efficiently and effectively in their scholarship. A sizable proportion of SLAC undergraduates pursue graduate study, so it is important to create and support an environment where students learn and internalize best research practices early in their careers. Students who pursue post-graduate work outside of academia also benefit from their development as critical consumers (and creators) of research through their coursework and beyond.

Recognizing and addressing challenges and opportunities SLAC faculty face as they contend with adopting open scientific practices in their classrooms and laboratories serve as the guiding framework of this paper. Although the conversation about accountability and transparency in psychological science often focuses on scholars at research institutions (R1s), there are arguably just as many - if not more - scholars grappling with open science practices at SLACs. One prominent professional organization, the Society for Personality and Social Psychology (SPSP), found between 50 and $60 \%$ of members worked at teaching-focused institutions (M. Leary, personal communication, July 27, 2018), and many of these faculty are engaging in timely conversations about research transparency.

SLAC faculty may be adopting open science practices at a slower pace than colleagues at $\mathrm{R} 1 \mathrm{~s}$. The benefits and costs to incorporating open science practices are not uniform and can differ, for instance, by career stage (Gernsbacher, 2018). Just as early career researchers face unique considerations regarding open science, so too do faculty at SLACs. Although voices from outside research universities enter into the conversation (cf. the Society for Improving Psychological Science [SIPS] has Executive Committee seats specifically designated for members at non-Ph.D.-granting institutions), our sense is that psychological scientists from research-intensive institutions comprise the preponderance of the voices in the open science conversation. Indeed, fewer than $5 \%$ of total attendees and $10 \%$ of faculty attendees at the SIPS 2018 Annual meeting were affiliated with SLACs. At the same time, faculty from liberal arts schools are beginning to think about the ways open science is enacted in their cultures, through 
SLAC-specific workshops ${ }^{1}$ and communities ${ }^{2}$, and greater focus at SIPS on working with undergraduates. We see enthusiasm for bringing open science into the liberal arts context, and we also see many colleagues facing challenges in their own teaching and research associated with some of the changes elicited by the move toward open science. These issues may be especially acute for junior colleagues as they work towards tenure and promotion. We hope this article will serve as advice for current and aspiring SLAC faculty and inform recommendations and expectations about doing open science in this context. Yes, times have changed, but with a commitment to understand and support one another in making these important adjustments, the future of open psychological science at SLACs is bright.

\section{Open Science Overview}

Several roughly contemporaneous events spurred psychology's transformation, including an article that claimed to provide evidence for precognition - colloquially known as a form of extrasensory perception (Bem, 2011) - in social psychology's premier outlet. The research underlying this claim, considered by some to be beyond the "cutoff for craziness" (Hofstadter, 2011), was criticized on statistical and methodological grounds (Wagenmakers, Wetzels, Borsboom, \& van der Maas, 2011) and for its failure to replicate (Galak, LeBoeuf, Nelson, \& Simmons, 2012).

While precognition stayed on the fringes, concerns grew about several well-established and influential programs of research (e.g., Carney, Cuddy, \& Yap, 2010; Doyen, Klein, Pichon, \& Cleeremans, 2012). The Open Science Collaboration's (Open Science Collaboration, 2015) replication project attempted to estimate replicability in psychology by marshalling researchers to conduct high-powered replications of 100 cognitive or social psychology studies that mirrored the original studies's methods as closely as possible. Using a conservative estimate of replicability, approximately two-thirds of studies did not replicate, suggesting a high prevalence of false positives in the psychological literature. The landscape is broader than psychology: In a survey of over 1500 social and natural scientists, over $90 \%$ of researchers reported that there was a reproducibility crisis, which was attributed to publication pressures and selective reporting of methods and analysis (Baker, 2016).

What practices lead to so many false positives? An influential paper (Simmons, Nelson, \& Simonsohn, 2011) introduced the idea of researcher degrees of freedom - the practice of making decisions during data collection and analysis that inflate the probability of making a Type I error above (often well above) 5\% (see also Gelman \& Loken, 2013). These practices termed p-hacking - include reporting results for only some dependent variables or conditions of independent variables, selectively excluding data, and checking data during collection and deciding whether to continue or terminate data collection. This work showed how questionable research practices (QRPs) could generate false positives in the literature. These practices were

${ }^{1}$ AALAC 2020 - Teaching and Employing Open Science Practices at Small Liberal Arts Colleges (https://osf.io/gs2ch/) [postponed due to covid]

2 The PUI Open Psychological Science (POPs) Network https://app.slack.com/client/TBKRR637U/CBLU9K99C. 
common and normative (John, Loewenstein, \& Prelec, 2012) and even encouraged by approaches that suggested that hypotheses should be driven by data (Bem, 2004; Kerr, 1998).

Although many of these issues were not new (Greenwald, 1976) and not limited to psychology (Baker, 2015), this confluence of events generated a unique sense of urgency, which in turn generated momentum toward open science, a set of values and practices characterized by “[t]ransparency, openness, and reproducibility" (Nosek et al., 2015).

\section{Teaching Open Science}

Even in the earliest days of the open science movement, scientists recognized the benefits of including undergraduates, and faculty are increasingly engaging in discussions regarding whether and how the replicability crisis and open science practices should be presented to students (Chopik, Bremner, Defever, \& Keller, 2018). We believe that the unique elements of liberal arts education lend themselves to the teaching of open science at SLACs (Strand \& Brown, 2019).

\section{Opportunities and Challenges}

Even faculty who regularly engage in best open science practices may be hesitant to engage undergraduates in discussions regarding the replicability crisis. Due to their high teaching loads and absence of graduate students to lead discussion sections, faculty might feel they lack time to add to their courses or to create a course that addresses the open science movement and practices. Faculty may fear students will lose trust in the field, or worry that they are not knowledgeable or qualified enough about open science to teach it.

However, features of SLACs make them ideal settings for engaging with open science. SLAC students generally have broad interests and have made the decision to further their education at an institution that values critical thinking and a well-rounded education. Small class sizes and interactive pedagogical approaches facilitate productive and robust discussions. Perhaps most notably, most SLACs value, or even demand, faculty-student research collaborations, with expectations that students will collaborate on published work (Reavis \& Thomas, 2019). Indeed, many SLACs purposely blur the line between teaching about research and doing research (Astin, 1999). SLAC undergraduates attend selective doctoral programs at high rates (Thomas R. Cech, 1999), so time spent training the next wave of researchers in open science practices is time well spent. Faculty often teach broadly beyond their narrow speciality, and can make use of helpful guides for getting started with open science (e.g., Kathawalla, Silverstein, \& Syed, 2020; Klein et al., 2018). The Open Scholarship Knowledge Base (https://www.oercommons.org/hubs/OSKB) is a repository of open science materials; as of this writing the "Teaching" section included 103 resources such as syllabi, class activities, readings, and lectures. Below we review different approaches to teaching open science and highlight additional available resources.

\section{Teaching Undergraduates Open Science}

At the introductory level, faculty might offer a standardized one-hour lecture (available at https://osf.io/mh9pe/) about replication and best practices (Chopik et al., 2018). Students who 
heard this lecture understood psychology's replications challenges but did not see this problem as limited to the field of psychology - they considered psychology to be similar to other fields in the natural sciences. Guest lectures from faculty within and outside one's institution can provide an introduction to open science - even for the trepidatious instructor, teaching open science to undergraduates might be as simple as phoning a friend or colleague. Further, given the wide-spread use of Zoom and other technologies, many SLAC faculty are likely to be comfortable with bringing virtual guest speakers into the classroom.

Faculty also can extend the coverage of open science with their own materials. The authors of this paper have created syllabi, PowerPoint presentations, and assignments addressing the main issues. We weave open science into all of our classes, frequently circling back to talk about replicability and reproducibility in terms of specific findings and open science more generally. Without exception, students are interested in the topic and able to understand the central issues. Many have heard about the replicability crisis, typically filtered by journalists, and are forming opinions about psychology research based on haphazard and often erroneous information (Chopik et al., 2018). Consequently, they appreciate having a richer understanding of the issues. One of us (BL) has taught an entire course on open science (https://osf.io/bpycq/), and other syllabi for open science classes are readily available (https://osf.io/vkhbt/). Furthermore, organizations like Project TIER (Teaching Integrity in Empirical Research; https://www.projecttier.org) support the development of pedagogies that promote transparency in the research process).

Students - with careful supervision - can conduct their own replications as part of their coursework. The process of conducting a replication demands that students think concretely about principles of research design such as study design and implementation, power and effect size, and dissemination that may be abstractions when only incorporated into class discussions (McKelvie \& Standing, 2018). Students gain meaningful research experience and generate a true contribution to the literature instead of a shortly-forgotten class assignment (Frank \& Saxe, 2012; Hawkins et al., 2018). At least one effort, the Collaborative Replications and Education Project (CREP; Wagge et al., 2019), facilitates crowd-sourced undergraduate replications that can culminate in publication.

Discussions of open science are especially rich in courses focusing on research methods and statistics. For example, collecting and analyzing data for a group project is a required component in one of our (JW) statistics and advanced methods courses, and students in one of our (KL) seminars conduct replications of one of the studies they read. Through preregistration (discussed in more detail below), students detail their research questions, hypotheses, methods, and data analytic procedures prior to the onset of data collection. In these courses students preregister their studies, which compels them to state their research questions, hypotheses, data collection procedures, and analysis plans clearly and succinctly (Blincoe \& Buchert, 2019). Students must understand statistical concepts to answer the preregistration questions relating to power and planned analytic procedures. Treating the preregistration template as a running document allows students to complete sections as they learn about them in class. Before data analysis begins, completed pre-registrations are reviewed and graded. 
Given SLAC's emphasis on meaningful research experience, it is not surprising that individual labs are a fertile opportunity for engaging students with open science. This context is the ideal place to model open science practices and find what works (and what doesn't) for your specific lab. Strand and Brown (2019) outline ways to include undergraduates in conducting reproducible research, and they found that a simple way to introduce open science to students in their labs is to read and discuss papers related to the replication crisis and QRPs. For those new to open science, designating a highly motivated student as the undergraduate in charge of becoming the lab open science "expert" can be a time-saver. Manuals that clearly lay out both open science principles and practices facilitate bringing open science into the lab (e.g., http://bit.ly/opensciencemanual). Students can participate in projects such as CREP (discussed earlier) or field-wide replication initiatives (two of our undergraduates were co-authors on the OSC Science paper).

Of course, not all students enrolling in psychology courses will go on to do research. We argue that learning about open science is equally important for students studying psychology as part of their liberal arts experience or general education requirements. Through thinking about open science and best research practices, students develop the transferable skills at the heart of a liberal arts education: Framing questions, thinking critically, working collaboratively, grappling with data, and communicating clearly.

\section{Open Science Practices}

\section{Preregistration}

The first step in the open science process is preregistration, which is a plan articulated before data collection in which investigators make concrete decisions about every aspect of the research process. It spells out methods, specific hypotheses, and a data analytic plan, and is uploaded to a secure, time-stamped, read-only repository (e.g., the Open Science Framework [OSF] from the Center for Open Science [COS], AsPredicted.org, socialscienceregistry.org, or clinicaltrials.gov). Thorough preregistration templates guide researchers through their data analysis, providing safeguards against QRPs such as hypothesizing after the results are known (Kerr, 1998) and p-hacking. Guides differ in their specificity, with more comprehensive plans requiring anticipated sample size, stopping rules for data collection, details about procedures, stimuli, and measures used in the study, and specific criteria for excluding data prior to analysis.

While this seems like a patently good thing, many faculty who were trained before the open science era learned to defy the conventional research process in favor of a "more exciting" approach. In The Compleat Academic (Darley, Zanna, Roediger, \& American Psychological Association, 2004), billed as a guide for beginning social scientists, Bem (2004) urged scientists to frame research in the best possible light after the fact: "Think of your dataset as a jewel. Your task is to cut and polish it, to select the facets to highlight, and to craft the best setting for it" (p. 188). Feeling smart enough to avoid the perils of this approach, psychology seemed to adopt Bem's heresy as gospel. This norm, together with pressure to publish sleek and surprising findings, contributed to psychology's replication crisis.

For some of us, giving up Bem's exciting approach to psychological research has been deflating. It takes precious time to catalogue every imperfect step of the research process, and 
demands on faculty at SLACs might slow them down even further. But this is what science needs: An explicit and transparent accounting of how we have made our discoveries. By becoming more process- than outcome- focused, we enable others to retrace a project's steps, which promotes systematic, incremental discovery. So, although preregistration takes time, it not only represents good science, it provides a concrete mechanism for teaching best practices in scientific inquiry. Preregistration as has become one of the cornerstones of open science practices (Nosek, Ebersole, DeHaven, \& Mellor, 2018; Nosek \& Lindsay, 2018), with over 10,000 projects preregistered in 2018 alone. Over the last half-decade the number of preregistrations has roughly doubled each year (Open Science Foundation, n.d.).

\section{Challenges \& Opportunities}

Preregistration templates serve as a starting point. We find that some templates can be inaccessible to those new to the process. McIntrye and Le (2018) (https://osf.io/n39tr/) constructed a preregistration template that balances thoroughness and accessibility with undergraduate researchers in mind. This model serves researchers at all career stages, including undergraduates and researchers who have little past experience with the preregistration process.

Preregistration is especially well-suited to undergraduate students as a pedagogical tool that provides an opportunity to assess their understanding of their research question, methods, and plans for data analysis. In short, it serves as a checkpoint during the research process letting the faculty supervisor evaluate if students understand their projects sufficiently to commence data collection (Blincoe \& Buchert, 2019). Faculty need to consider how well this process scales from a handful of students in a lab class to having a larger class do preregistration. While we certainly recommend having thesis students preregister their projects, it may not be feasible to have all students in larger courses do full preregistrations.

\section{Research Design and Sample Size}

The habit of preregistering studies likely creates a shift in thinking about sampling and study design, which are critical components of the preregistration process.

Sample Size. Psychology relies heavily on small samples. For a very long time, we have known that these small samples do not get the job done. Cohen found that studies published in the 1960 volume of the Journal of Abnormal and Social Psychology had a mean power to detect medium-sized effects of only .48 (Cohen, 1962) - worse than a flip of a coin.

Old habits die hard, and researchers have done too little over the decades to increase the statistical power of our studies (Cohen, 1992). In the open science era, researchers (including those at SLACs) will have to answer the renewed, better-informed call for increased sample sizes. Many faculty, especially those at SLACs, conduct small sample studies because of significant constraints that limit participant recruitment. This choice is often understandable - we urge researchers to make such decisions in the context of open science, the risks of underpowered studies, and the potential for $p$-hacking. Part of the solution is to err on the side of larger samples, and the other part is to design better studies (discussed below). A priori power analyses provide evidence-based guidance for sample size. It is fine to balance power considerations against the feasibility of conducting larger studies and to make research decisions 
ahead of time that reflect this trade-off and seek to increase power and protect against false positives through forthright and better study design.

Study Design. While larger sample sizes are the most obvious way to increase power and reduce the influence of "researcher degrees of freedom" in producing false positives, a welldesigned study - clearly conceptualized at the preregistration stage - takes some of the burden off sample size. Distinct and robust experimental manipulations, reliable and valid dependent measures, and (where appropriate) within-subjects designs increase statistical power. Likewise, carefully pre-selected (and preregistered) covariates can account for variance in a dependent variable and increase power. Even so, it is important to keep in mind Cohen's (1990) note of caution when it comes to "throwing together" your study materials:

One thing I learned over a long period of time...is the validity of the general principle that less is more, except of course for sample size. I have encountered too many studies with prodigious numbers of dependent variables, or with what seemed to me far too many independent variables, or (heaven help us) both. In any given investigation that isn't explicitly exploratory, we should be studying few independent variables and even fewer dependent variables ....Using the .05 level for many tests...greatly increases the chances of discovering things that aren't so. (p. 1304)

\section{Challenges and Opportunities}

Conducting larger studies might seem daunting. Most SLACs have limited participant pools, and researchers can quickly deplete their available participants and/or funds when trying to increase their sample size. Participant compensation costs might be prohibitive, especially because grant support is less common at SLACs. This might increase the challenge of obtaining publishable data from any particular study and have a disproportionate effect on researchers at SLACs, where research already moves more slowly than at larger universities.

These are significant challenges, but they also present opportunities that can benefit researchers at SLACs. Open science levels the playing field for studies that are well-conceived, and the slower pace and hands-on role of faculty at SLACs lend themselves to honing welldesigned studies. In other words, researchers should not "compete" in terms of quantity; they should focus instead on the research process and strive for higher quality studies and lines of research. Registered reports, in which journal articles are reviewed and accepted (or not) based on a literature review and preregistered methodology and analytical plans, might be attractive to SLAC faculty in helping to mitigate the effects of the elongated timeline from idea to publication (Nosek \& Lakens, 2014).

SLAC faculty can employ many strategies to gather more data for their well-designed studies. Researchers can collaborate with colleagues at other institutions and find ways to share participants. Several mechanisms, such as the Psychological Science Accelerator (Moshontz et al., 2018) or Study Swap (StudySwap: A Platform for Interlab Replication, Collaboration, and Research Resource Exchange, 2016) facilitate collaborations, crowdsourcing, and matching researchers' needs with available resources. Similarly, they can conduct efficient large-sample studies online through services such as Amazon's mTurk or Prolific, followed by focused replications in the laboratory. Reliance on Internet samples presents its own challenges - it may 
be less appropriate for researchers in developmental or abnormal psychology than for those in cognitive or social psychology, and it constrains the kinds of methods that can be used. But for some types of research, the Internet can be a useful resource when conducting larger studies.

Before open science practices were widely adopted, researchers often looked at data while a study was in progress to decide whether to continue or terminate data collection (John et al., 2012). This practice, which inflates Type I errors, still retains practical allure: SLAC researchers could easily use a semester's worth of participants just to discover an effect size of $d$ $=0.00$. We would rather discover that after our 30th than 300th participant! Although concerns about p-hacking argue against constantly peeking at one's data, open science provides an opportunity for accomplishing this goal more transparently by conducting analyses at predetermined intervals throughout the data collection process using sequential analysis (Lakens, 2014), which balances efficient use of available subjects with mitigated risk of Type I error.

\section{Materials and Data Sharing}

Open science requires transparency throughout the entire research process. Sharing materials and data increases the trustworthiness of a research project and makes it possible for others to replicate our work. Sharing data requires greater accountability by researchers, who must demonstrate that they have handled the data properly, used data analysis tools adeptly, and did not overlook potential alternative explanations for their findings. The scrutiny that accompanies open science can begin to feel a little like inviting other researchers to look at how you've organized your bedroom closet. Yes, you wear those clothes in public, but you may not be proud of how you arrange your wardrobe and not every garment is part of your outerwear.

\section{Challenges and Opportunities}

Sharing materials and data can feel even more revealing for faculty at SLACs, where materials are designed with undergraduates, participants are difficult to recruit, and data analysis is done without the helpful eyes of graduate students or post-docs. Being willing to share materials and data with the broader academic community requires good faith and courage. This good faith is undermined, in part, when a colleague who can churn out multiple studies a month at a big institution is able to "scoop" the next step in your line of research. The OSF allows researchers to keep things under wraps by letting them upload and time-stamp materials but embargo them until a later point in time. Materials may also be shared upon request, or only with certain individuals, such as collaborators or the manuscript reviewers.

Open science means open data - which also means risks associated with data sharing (Meyer, 2018). These risks may be exacerbated at SLACs, where maintaining participant anonymity in shared data could be a challenge. If participants include undergraduates from one's campus, it is likely they could be identified even when information such as names and email addresses is withheld. When there are fewer participants drawn from a small population, sharing data related to race and ethnicity or including qualitative data and first-person responses to experimental prompts runs the risk of linking a set of responses to one particular person (Mackinnon, 2014; Rocher, Hendrickx, \& de Montjoye, 2019). 
Researchers can balance concerns about privacy with the desire to share data in order to reap some of the benefits of sharing materials and data (Meyer, 2018). For example, posted data can be encrypted, and the corresponding author can create a required data sharing agreement focused on protecting participants' confidentiality. Further, they might omit certain demographic data or other potentially identifying responses from the data they share. In certain cases it may make sense to have data available selectively (to reviewers, for example), in summary form (e.g., for future meta-analyses) or even not at all. The researcher would need to note their rationale for why they were not able to make the data public. Openly reflecting on the benefit-to-risk ratio is another form of transparency. R packages such as anonymizer or sdcMicro can generate anonymized data sets that protect participant confidentiality.

Researchers at SLACs can also reap benefits of sharing materials and data. Using a platform like the OSF as a tool to organize research projects can be incredibly useful. SLAC faculty often collaborate with colleagues at other institutions. Having a centralized location (with free storage!) to hold one's hypotheses, materials, data, analyses, and documents greatly enhances the opportunity for effective collaboration. But sharing materials and data is useful not just to increase efficiencies and effectiveness in working with other researchers. Systematically tracking, organizing, and sharing materials and data also make for more productive collaborations with current and future generations of undergraduate students who carry out research in your classes or work in your research lab. Current students learn best practices in open science as they organize and share materials and data. And as this becomes a routine practice, future students will be able to learn from what came before. They will have ready access to materials that were used in a study many years ago. This saves the research mentor time and energy that would otherwise be expended on tracking down questionnaires, data sets, and analyses. In our experience, the OSF has created a platform that allows for maximal flexibility in how you would like to manage and share materials and data. Further, OSF gives unconstrained free storage. You can take everything with you over time, even if you move from one institution to another. The benefits, in our opinion, outweigh the risks discussed earlier.

Beyond open materials and data, sharing the code underlying the data analysis is becoming increasingly normative as a means of supporting computational reproducibility of results. This can easily be accomplished in R, but is also achieved in SPSS by "pasting" and saving syntax. Not only does providing code/syntax facilitate computational reproducibility, but it provides interesting teaching opportunities; for example, in one of our labs (BL), it has become normative for students to verify each others' results from annotated SPSS syntax that accompanies their data and results before research papers are completed.

\section{Professional Development Considerations}

\section{Hiring and Evaluation}

The growing normalization of open science practices for early career researchers leads to important considerations when hiring and evaluating faculty for tenure and promotion (Gernsbacher, 2018). These issues can be magnified in smaller departments due to idiosyncrasies in hiring and promotion that are inherent within SLAC contexts. Colleges, and departments within those institutions, need to explicitly align incentives with their ideals and values for open science to take hold in faculty research and teaching. 


\section{Considerations for Hiring}

At research universities much of the momentum in open science is driven by graduate students. At smaller undergraduate-focused institutions, new hires and senior faculty who embrace these new developments will likely lead this progress. Given the relative infrequency of hires in a small department, it may take a number of years to change a department's orientation towards open science strictly through recruiting new faculty. However, depending on the lifecycle of a small department, it may just take a handful of new hires in a short period of time to change departmental culture.

When recruiting applicants, committees may make open science engagement an explicit criteria during the search (e.g., requiring candidates to speak to these issues in their applications). However, this approach can be problematic by putting an additional filter in place that, in conjunction with other desired criteria (e.g., desire to work in a liberal arts environment, inclusivity/diversity considerations), can limit the applicant pool. This filter may restrict the applicant pool in some subdisciplines more than others (e.g., clinical and developmental psychology, which to date have not adopted open science practices as quickly as other subdisciplines). A more realistic and effective approach may be to signal to applicants explicitly that the department values research openness and transparency within the job advertisement or on the departmental website, without formally making it a criteria used to evaluate candidates. In short, search committees should think carefully about how they value open science relative to other criteria, understand how this may impact the applicant pool, and be strategic in how this is communicated to potential applicants.

Additionally, search committees need to understand the changing landscape of scholarship. The first step is to educate committee members on new forms of publication, such as open access journals, registered reports, and preprints. For example, while open access journals allow dissemination of research beyond those with (expensive, and sometimes, for SLACs, costprohibitive) journal subscriptions, existing journals are known entities, and metrics such as impact factor crudely, if imperfectly, quantify their quality in ways that are understood by hiring committees. SLAC faculty are small, and during hiring and evaluation processes may lack appropriate expertise to evaluate a candidate's work. Therefore, they may be more likely to use journal impact factors or perceived prestige as a proxy for quality of work. We suggest that candidates - and their letter-writers - educate potential and current colleagues about open access. Search committees and tenure evaluators distant from candidates' intellectual approaches will need to rely more heavily on these external assessments. Arguably, changes brought about by open science are aligned with a more accurate process, in which work is evaluated on its merits rather than through the use of a heuristic about its outlet.

In addition, committees cannot solely look at number of publications on candidates' $\mathrm{CVs}$, since those engaging in open science practices may have fewer publications due to the extra time and resources involved in this approach and an absence of publications derived from p-hacking. Committees need to be open-minded and expansive in how they evaluate candidates' publication history if the goal is to promote open science within their departments.

\section{Considerations for Evaluation for Tenure and Promotion}


Beyond recruiting and hiring new colleagues who work from an open science perspective, educating others about and promoting open science is critical when it comes time to evaluate colleagues for renewal, tenure, and/or promotion. Senior members of a department can actively support open science by educating colleagues about the value of open science, and they can contextualize the quantity and timing of that work in their internal letters of support for their junior colleagues. One especially effective strategy may be to highlight the connection between open science and pedagogy, commenting on how students' educations are uniquely enhanced due to what they have learned about these "cutting edge" developments in the field.

If given the opportunity to suggest external letter writers for a colleague's promotion case, senior colleagues can make sure to list reviewers who understand open science and guide the committee away from external reviewers who are known to be hostile towards open science. Similarly, when asked to serve as an external letter writer, senior scholars can support open science by positively commenting on the value of open science practices and highlighting these aspects of the candidate's work when evaluating colleagues at other institutions.

\section{Participation in the Open Science Community}

Working in a small department can be isolating (Punt, 2017; Sandquist, Romberg, \& Yancey, 2013). Similarly, in a small department, one might be the only researcher, or among a small handful of researchers, interested in employing open science practices. Therefore, connecting to the wider open science community is critical for those at small schools wanting to deepen their engagement with open science.

Much of the day-to-day conversations about open science occur on social media. There is a vibrant open science community on Twitter, revolving around membership in SIPS (@improvingpsych) and their annual conference (e.g., \#SIPS2020), as well as stemming from the support for and use of the Center for Open Science (COS; @ OSFramework). Similarly, there are active groups on Facebook for discussing open science and methodological reform in psychology (e.g., the Psychological Methods Discussion Group). The social media focus of open science communication may be a double-edged sword when it comes to working in a small department at a teaching-focused institution. Faculty who spend more time in the classroom and less time in front of their computers may have trouble keeping up with the fast-paced open science conversations on social media. However, having these conversations take place online can level the playing field, giving those at small and large institutions an equal chance to participate in the discussions and have their voices amplified.

In addition to, or instead of, participating in the open science communities on social media, there are opportunities to connect with colleagues at open science-focused conferences, such as the annual meeting of SIPS. SIPS is a summer conference with reasonable registration fees, which hopefully will increase the likelihood that those at smaller institutions can attend. A unique aspect of SIPS is that there is not a submission process to present at the conference, and in fact, very few presentations are included in the conference schedule at all. Instead, the sessions, which feature interactive "hack-a-thons" and "unconferences," evolve organically over the course of the conference based on mutual interests or project goals. This format means that teaching-focused sessions and conversations between researchers at similar institutions can form easily at the conference, with a critical mass of participants. Finally, inclusion is a primary focus 
of SIPS, and the organization is structured to make sure that those at non- $\mathrm{PhD}$ granting institutions have a strong voice in its governance, and most projects stemming from work at SIPS are reviewed with respect to many forms of diversity, including type and size of institution.

Beyond seeking out colleagues on social media or at SIPS, open science provides an opportunity to connect with colleagues in other small departments, which is especially important when you do not have many colleagues who share a common disciplinary background. A lack of close colleagues in one's research area can feel isolating and limit opportunities for collaboration and intellectual sharing. However, because open science cuts across sub-disciplines, it can serve as a central unifying force in a small department, giving a platform for colleagues with diverse research backgrounds to work together from a common perspective.

\section{Conclusion}

Although the obstacles to adopting open science practices might seem considerable, many resources exist to help SLAC faculty transition to teaching and practicing open science. This article references many of those resources and includes many SLAC-specific suggestions, including tips for preregistering studies, data sharing, and teaching open science to undergraduates. Although practicing open science requires some work on the front end, we firmly believe that adopting and teaching these practices will make the field of psychology stronger while providing exceptional training for undergraduates and facilitating SLAC faculty's research agendas.

Open science is consistent with SLACs' pedagogical and research goals. For example, requiring that undergraduate students preregister studies ensures that they fully understand key components of doing research. Material and data sharing empower faculty collaboration and access to larger samples. In addition, the open science community seeks to support and empower early career researchers and provides a variety of resources to this end. As more and more researchers adopt open science practices, one can expect that open science considerations will become a part of hiring and tenure and promotion processes. The open science movement is not a phase - it represents the direction that science is moving. Despite the challenges, faculty at small liberal arts colleges have much to gain by learning, doing, and teaching open science. 


\section{References}

Astin, A. (1999). Student involvement: A developmental theory for higher education. Journal of College Student Development, 40(5), 518-529.

Baker, M. (2016). 1,500 scientists lift the lid on reproducibility. Nature News, 533(7604), 452. https://doi.org/10.1038/533452a

Bem, D. J. (2004). Writing the empirical journal article. In J. M. Darley \& M. P. Zanna (Eds.), The Compleat Academic: A Practical Guide for the Beginning Social Scientist (2nd ed., pp. 185-219). Washington, D. C.: American Psychological Association.

Bem, D. J. (2011). Feeling the future: Experimental evidence for anomalous retroactive influences on cognition and affect. Journal of Personality and Social Psychology, 100(3), 407-425. https://doi.org/10.1037/a0021524

Blincoe, S., \& Buchert, S. (2019). Research Preregistration as a Teaching and Learning Tool in Undergraduate Psychology Courses. Psychology Learning \& Teaching, 1475725719875844. https://doi.org/10.1177/1475725719875844

Carney, D. R., Cuddy, A. J., \& Yap, A. J. (2010). Power posing: Brief nonverbal displays affect neuroendocrine levels and risk tolerance. Psychological Science, 21(10), 1363-1368. https://doi.org/10.1177/0956797610383437

Chopik, W. J., Bremner, R. H., Defever, A. M., \& Keller, V. N. (2018). How (and whether) to teach undergraduates about the replication crisis in psychological science. Teaching of Psychology, 45(2), 158-163. https://doi.org/10.1177/0098628318762900

Cohen, J. (1962). The statistical power of abnormal-social psychological research: A review. The Journal of Abnormal and Social Psychology, 65(3), 145-153. https://doi.org/10.1037/h0045186

Cohen, J. (1990). Things I have learned (so far). American Psychologist, 45(12), 1304-1312. https://doi.org/10.1037/0003-066X.45.12.1304

Cohen, J. (1992). Statistical power analysis. Current Directions in Psychological Science, 1(3), 98-101. https://doi.org/10.1111/1467-8721.ep10768783

Darley, J. M., Zanna, M. P., Roediger, H. L., \& American Psychological Association (Eds.). (2004). The compleat academic: A career guide (2nd ed). Washington, DC: American Psychological Association.

Doyen, S., Klein, O., Pichon, C.-L., \& Cleeremans, A. (2012). Behavioral priming: It's all in the mind, but whose mind? PloS One, 7(1), e29081. https://doi.org/10.1371/journal.pone.0029081

Fanelli, D. (2018). Opinion: Is science really facing a reproducibility crisis, and do we need it to? Proceedings of the National Academy of Sciences, 115(11), 2628-2631. https://doi.org/10.1073/pnas.1708272114

Frank, M. C., \& Saxe, R. (2012). Teaching replication. Perspectives on Psychological Science, 7(6), 600-604. https://doi.org/10.1177/1745691612460686

Galak, J., LeBoeuf, R. A., Nelson, L. D., \& Simmons, J. P. (2012). Correcting the past: Failures to replicate psi. Journal of Personality and Social Psychology, 103(6), 933-948. https://doi.org/10.1037/a0029709

Gelman, A., \& Loken, E. (2013). The garden of forking paths: Why multiple comparisons can be a problem, even when there is no "fishing expedition" or "p-hacking" and the research hypothesis was posited ahead of time. Department of Statistics, Columbia University. 
Retrieved from

http://www.stat.columbia.edu/ gelman/research/unpublished/p_hacking.pdf

Gernsbacher, M. A. (2018). Rewarding research transparency. Trends in Cognitive Sciences, 22(11), 953-956. https://doi.org/10.1016/j.tics.2018.07.002

Greenwald, A. G. (1976). An editorial. Journal of Personality and Social Psychology, 33(1), 17.

Hawkins, R. X. D., Smith, E. N., Au, C., Arias, J. M., Catapano, R., Hermann, E., ... Frank, M. C. (2018). Improving the replicability of psychological science through pedagogy. Advances in Methods and Practices in Psychological Science, 1(1), 7-18. https://doi.org/10.1177/2515245917740427

Hofstadter, D. (2011, January 6). A cutoff for craziness. Retrieved June 30, 2019, from https://www.nytimes.com/roomfordebate/2011/01/06/the-esp-study-when-science-goespsychic/a-cutoff-for-craziness

John, L. K., Loewenstein, G., \& Prelec, D. (2012). Measuring the prevalence of questionable research practices with incentives for truth telling. Psychological Science, 23(5), 524532. https://doi.org/10.1177/0956797611430953

Kathawalla, U.-K., Silverstein, P., \& Syed, M. (2020). Easing into open science: A guide for graduate students and their advisors [Preprint]. PsyArXiv. https://doi.org/10.31234/osf.io/vzjdp

Kerr, N. L. (1998). HARKing: Hypothesizing after the results are known. Personality and Social Psychology Review, 2(3), 196-217. https://doi.org/10.1207/s15327957pspr0203_4

Klein, O., Hardwicke, T. E., Aust, F., Breuer, J., Danielsson, H., Hofelich Mohr, A., ... Frank, M. C. (2018). A practical guide for transparency in psychological science. Collabra: Psychology, 4(1), 20. https://doi.org/10.1525/collabra.158

Lakens, D. (2014). Performing high-powered studies efficiently with sequential analyses. European Journal of Social Psychology, 44(7), 701-710. https://doi.org/10.1002/ejsp.2023

Mackinnon, S. (2014, July 9). Privacy in the age of open data. Retrieved from http://osc.centerforopenscience.org/author/sean-mackinnon.html

McIntyre, K., \& Le, B. (2018). OSL/PT Preregistration Template. https://doi.org/10.17605/OSF.IO/N39TR

McKelvie, S., \& Standing, L. G. (2018). Teaching psychology research methodology across the curriculum to promote undergraduate publication: An eight-course structure and two helpful practices. Frontiers in Psychology, 9. https://doi.org/10.3389/fpsyg.2018.02295

Meyer, M. N. (2018). Practical tips for ethical data sharing. Advances in Methods and Practices in Psychological Science, 1(1), 131-144. https://doi.org/10.1177/2515245917747656

Moshontz, H., Campbell, L., Ebersole, C. R., IJzerman, H., Urry, H. L., Forscher, P. S., ... Chartier, C. R. (2018). The Psychological Science Accelerator: Advancing psychology through a distributed collaborative network. Advances in Methods and Practices in Psychological Science, 1(4), 501-515. https://doi.org/10.1177/2515245918797607

Nelson, L. D., Simmons, J., \& Simonsohn, U. (2018). Psychology's renaissance. Annual Review of Psychology, 69, 511-534. https://doi.org/10.1146/annurev-psych-122216-011836

Nosek, B. A., Alter, G., Banks, G. C., Borsboom, D., Bowman, S. D., Breckler, S. J., ... Yarkoni, T. (2015). Promoting an open research culture. Science, 348(6242), 1422-1425. https://doi.org/10.1126/science.aab2374 
Nosek, B. A., Ebersole, C. R., DeHaven, A. C., \& Mellor, D. T. (2018). The preregistration revolution. Proceedings of the National Academy of Sciences, 115(11), 2600-2606. https://doi.org/10.1073/pnas.1708274114

Nosek, B. A., \& Lakens, D. (2014). Registered reports: A method to increase the credibility of published results. Social Psychology, 45(3), 137-141. https://doi.org/10.1027/18649335/a000192

Nosek, B. A., \& Lindsay, D. S. (2018). Preregistration becoming the norm in psychological science. APS Observer.

Open Science Collaboration. (2015). Estimating the reproducibility of psychological science. Science, 349(6251), aac4716. https://doi.org/10.1126/science.aac4716

Open Science Foundation. (n.d.). OSF Registries improves transparency and rigor of research. Retrieved June 6, 2020, from OSF Registries improves transparency and rigor of research website: https://www.cos.io/our-products/osf-registries

Punt, J. (2017). A career at a small liberal arts college. Cold Spring Harbor Perspectives in Biology, 9(10), a032789. https://doi.org/10.1101/cshperspect.a032789

Reavis, R. D., \& Thomas, M. A. (2019). Building a Scaffolded Research Experience for Undergraduates. Frontiers in Psychology, 10. https://doi.org/10.3389/fpsyg.2019.00524

Rocher, L., Hendrickx, J. M., \& de Montjoye, Y.-A. (2019). Estimating the success of reidentifications in incomplete datasets using generative models. Nature Communications, 10(1), 1-9. https://doi.org/10.1038/s41467-019-10933-3

Sandquist, J., Romberg, L., \& Yancey, P. (2013). Life as a professor at a small liberal arts college. Molecular Biology of the Cell, 24(21), 3285-3291. https://doi.org/10.1091/mbc.e13-06-0341

Simmons, J. P., Nelson, L. D., \& Simonsohn, U. (2011). False-positive psychology: Undisclosed flexibility in data collection and analysis allows presenting anything as significant. Psychological Science, 22(11), 1359-1366. https://doi.org/10.1177/0956797611417632

Strand, J. F., \& Brown, V. A. (2019). Publishing open, reproducible research with undergraduates. Frontiers in Psychology, 10. https://doi.org/10.3389/fpsyg.2019.00564

StudySwap: A platform for interlab replication, collaboration, and research resource exchange. (2016). Retrieved from https://osf.io/9aj5g/

Thomas R. Cech. (1999). Science at liberal arts colleges: A better education? Daedelus, 128(1), 195-197.

Vazire, S. (2018). Implications of the credibility revolution for productivity, creativity, and progress. Perspectives on Psychological Science, 13(4), 411-417. https://doi.org/10.1177/1745691617751884

Wagenmakers, E.-J., Wetzels, R., Borsboom, D., \& van der Maas, H. L. J. (2011). Why psychologists must change the way they analyze their data: The case of psi: Comment on Bem (2011). Journal of Personality and Social Psychology, 100(3), 426-432. https://doi.org/10.1037/a0022790

Wagge, J. R., Brandt, M. J., Lazarevic, L. B., Legate, N., Christopherson, C., Wiggins, B., \& Grahe, J. E. (2019). Publishing research with undergraduate students via replication work: The Collaborative Replications and Education Project. Frontiers in Psychology, 10. https://doi.org/10.3389/fpsyg.2019.00247 